\title{
Breath Test
}

National Cancer Institute

\section{Source}

National Cancer Institute. Breath Test. NCI Thesaurus. Code C116515.

Any test performed on air generated by the act of exhaling. 